\title{
LOCAL TIME AT FICTITIOUS STATES
}

\author{
BY DAVID WILLIAMS
}

Communicated by Henry McKean, Feb. 6, 1967

Theorems 1 and 2 will be contained in a forthcoming paper by the author. Theorem 1 follows from work of Ray [6] and Neveu [4], [5] but a new proof will be presented. Theorem 2 is a consequence of a result already announced in Williams [7]. The terminology and notation are as in Dynkin's book [1].

Let $E$ be a countable set and let $\left\{p_{i j}(t): i, j \in E ; t \geqq 0\right\}$ be a transition function on $E$ with the properties:

$$
\sum_{j \in E} p_{i j}(t)=1 ; \quad \lim _{u \downarrow 0} p_{i i}(u)=1 \quad(i \in E ; t \geqq 0) .
$$

Theorem 1. There exist a complete metric space $E^{+}$in which $E$ is dense and a strong Feller, stochastically continuous transition function $P(t)$ on $E^{+}$such that the following statements are true:

$$
\begin{aligned}
P(t, i,\{j\}) & =p_{i j}(t) \quad(i, j \in E ; t \geqq 0) ; \\
P(t, y, E) & =1 \quad\left(y \in E^{+} ; t>0\right) ;
\end{aligned}
$$

(iii) every Markov chain on $E$ with transition function $\left\{p_{i j}(t)\right\}$ has a right-continuous, strong Markov version taking values in $E^{+}$and with transition function $P(t)$.

For this version, limits from the left may not always exist in $E^{+}$and the property of quasi-left-continuity may not hold.

Let $\{x(t, \omega): t \geqq 0 ; \omega \in \Omega\}$ be some fixed right-continuous, strong Markov process on $E^{+}$with transition function $P(t)$. Let $x$ be a fixed point of $E^{+} \backslash E$ and define

$$
\begin{aligned}
T(\omega) & =\infty \quad \text { if } x(t, \omega) \neq x \quad \text { for all } t>0, \\
& =\inf \{t: t>0, x(t, \omega)=x\} \quad \text { otherwise. }
\end{aligned}
$$

Suppose that $x$ is such that

$$
\begin{gathered}
P_{x}\{T=0\}=1 ; \\
P_{\mu}\{T<\infty\}>0,
\end{gathered}
$$

$\mu$ being the initial distribution. ${ }^{1}$ Then local time at $x$ may always be defined by an equation similar to equation (1) below.

However, in order that the result may be stated in a form with the

\footnotetext{
${ }^{1}$ In current terminology, $x$ is neither polar nor semipolar.
} 
most direct intuitive content, it is assumed throughout the remainder of this note that $\{x(t, \omega)\}$ is irreducible recurrent, i.e., that, for $i, j \in E$ and $t>0$,

$$
p_{i j}(t)>0 \text { and } \int_{0}^{\infty} p_{i j}(u) d u=\infty .
$$

Let $\pi$ be the positive invariant measure on $E$ :

$$
\sum_{i \in E} \pi_{i} p_{i j}(t)=\pi_{j} \quad(j \in E ; t \geqq 0) .
$$

(Recall that $\pi$ is determined uniquely up to scalar multiples.)

For $j$ in $E$, introduce the normalized local time at $j$ :

$$
L(j, t ; \omega)=m\{s: 0 \leqq s \leqq t, x(s, \omega)=j\} / \pi_{j},
$$

$m$ denoting Lebesgue measure.

Theorem 2. There exist a sequence $\Delta=\left\{\delta_{n}\right\}$ with $\delta_{n} \downarrow 0$ and a set $\Omega_{0} \subseteq \Omega$ with $P_{\mu}\left\{\Omega_{0}\right\}=1$ such that, if $\omega \in \Omega_{0}$, then the limit

$$
L(x, t ; \omega)=\lim _{\Delta \in \delta \downarrow 0} \sum_{j \in E} P(\delta, x,\{j\}) L(j, t ; \omega)
$$

exists uniformly on all intervals of the form $\left[0, t_{0}\right]$. Furthermore, if the Markov time $\tau(t)$ is defined by the equation

$$
\tau(t)=\inf \{s: s+L(x, s ; \omega)>t\}
$$

then $z(\cdot)=x(\tau(\cdot))$ is a strong Markov process with minimal state-space $E \cup\{x\}$ and the point $x$ is an instantaneous state of $z(\cdot)$.

In view of the fact that $P(t)$ is stochastically continuous, Theorem 2 may be regarded as the appropriate version for Markov chains of the equation

$$
\lim _{j \rightarrow x} L(j, t ; \omega)=L(x, t ; \omega) \quad(t \geqq 0)
$$

which might possibly be expected to hold because of analogous results for certain diffusion processes. See Meyer [3] and the papers referred to therein for recent work on the existence and continuity of local time. Examples show that equation (2) is most decidedly false for certain Markov chains.

Lévy's well-known construction for local time (see for example $[2, \S I I I])$ would seem less direct than that afforded by equation (1) and appears slightly more difficult to justify in the situation considered here. The difficulty lies in the fact that one does not know $a$ priori of the existence of $z(\cdot)$. 
Problem. May the equation

$$
L(x, t ; \omega)=\lim _{\delta \downarrow 0} \sum_{j \in B} P(\delta, x,\{j\}) L(j, t ; \omega)
$$

be used to define local time simultaneously at all fictitious states?

\section{REFERENCES}

1. E. B. Dynkin, Theory of Markov processes, (English translation by J. Fabius, V. Greenberg, A. Maitra, G. Majone), Springer, Berlin, 1965.

2. P. Lévy, Processus semi-markovien, Proc. Internat. Congr. Math. Amsterdam 1954, pp. 416-426.

3. P. A. Meyer, Sur les lois de certaines fonctionnelles additives; applications aux temps locaux, Publ. Inst. Statist. Univ. Paris, 15 (1966), 295-310.

4. J. Neveu, Sur les états d'entrée et les états fictifs d'un processus de Markov, Ann. Inst. H. Poincaré 17 (1962) 323-337.

5. - Entrance, exit and fictitious states for Markoy chains, Proc. Aarhus Colloq. Combinatorial Probability 1962, pp. 64-68.

6. D. Ray, Resolvents, transition functions and strongly Markovian processes, Ann. of Math. 70 (1959), 43-72.

7. D. Williams, On local time for Markov chains, Bull. Amer. Math. Soc. 73 (1967), 432-433.

Clare College, Cambridge, England 